\title{
Latent Semantic Analysis using a Dennis Coefficient for English Sentiment Classification in a Parallel System
}

\author{
V.N. Phu, V.T.N. Tran
}

\author{
Vo Ngoc Phu* \\ Institute of Research and Development, \\ Duy Tan University-DTU \\ Da Nang, Vietnam \\ *Corresponding author: vongocphu03hca@gmail.com; vongocphu@dtu.edu.vn
}

\author{
Vo Thi Ngoc Tran \\ School of Industrial Management (SIM), \\ Ho Chi Minh City University of Technology - HCMUT, \\ Vietnam National University \\ Ho Chi Minh City, Vietnam \\ vtntran@hcmut.edu.vn
}

\begin{abstract}
We have already survey many significant approaches for many years because there are many crucial contributions of the sentiment classification which can be applied in everyday life, such as in political activities, commodity production, and commercial activities. We have proposed a novel model using a Latent Semantic Analysis (LSA) and a Dennis Coefficient (DNC) for big data sentiment classification in English. Many LSA vectors (LSAV) have successfully been reformed by using the DNC. We use the DNC and the LSAVs to classify 11,000,000 documents of our testing data set to 5,000,000 documents of our training data set in English. This novel model uses many sentiment lexicons of our basis English sentiment dictionary (bESD). We have tested the proposed model in both a sequential environment and a distributed network system. The results of the sequential system are not as good as that of the parallel environment. We have achieved $88.76 \%$ accuracy of the testing data set, and this is better than the accuracies of many previous models of the semantic analysis. Besides, we have also compared the novel model with the previous models, and the experiments and the results of our proposed model are better than that of the previous model. Many different fields can widely use the results of the novel model in many commercial applications and surveys of the sentiment classification.

Keywords: English sentiment classification; parallel system; Cloudera; Hadoop Map and Hadoop Reduce; Dennis Measure; Latent Semantic Analysis
\end{abstract}

\section{Introduction}

In this survey, our novel model is performed as follows: Firstly, we use the Dennis Coefficient (DNC) to identify the valences and polarities of the sentiment lexicons of the basis English sentiment dictionary (bESD). Then, the Latent Semantic Analysis (LSA) is improved by using the sentiment lexicons. All the positive documents of the training data set are transferred into one LSAV, called the positive LSAV group. All the negative documents of the training data set are transferred into one LSAV, called the negative LSAV group. Each document in the documents of the testing data set is transferred into one LSAV. We use the DNC to cluster this LSAV into either the positive LSAV group or the negative LSAV group. One similarity measure between the LSAV and the positive LSAV group is calculated certainly, called Measure_1 and one similarity measure between the LSAV and the negative LSAV group is calculated certainly, called Measure_2. If Measure_1 is greater than Measure_2, it means that the LSAV being close to the positive LSAV group is greater than the LSAV being close to the negative LSAV group, so 
the LSAV (corresponding to the document of the testing data set) is clustered into the positive. If Measure_1 is less than Measure_2, it means that the LSAV being close to the positive LSAV group is less than the LSAV being close to the negative LSAV group, so the LSAV (corresponding to the document of the testing data set) is clustered into the negative. If Measure 1 is as equal as Measure_2, it means that the LSAV being close to the positive LSAV group is as equal as the LSAV being close to the negative LSAV group, so the LSAV (corresponding to the document of the testing data set) is not clustered into both the positive and the negative. The LSAV is clustered into the neutral polarity. Therefore, the sentiment classification of this document is identified successfully. Finally, the sentiment classification of all the document of the testing data set is identified fully.

We firstly implement all the above things in the sequential system, and then, we perform all the above things in the parallel network environment to shorten the execution times of the proposed model.

Our proposed model has the crucial contributions to many areas of research as well as commercial applications as follows: (1) Many surveys and commercial applications can use the results of this work in a significant way; (2) The algorithms are built in the proposed model; (3) This survey can certainly be applied to other languages easily; (4) The algorithm of data mining is applicable to semantic analysis of natural language processing; (5) Millions of English documents are successfully processed for emotional analysis; (6) Our proposed model can be applied to many different parallel network environments such as a Cloudera system; (7) This study can be applied to many different distributed functions such as Hadoop Map (M) and Hadoop Reduce (R); (8) The LSA - related algorithms are proposed in this survey; (9) The DNC - related algorithms are built in this work.

\section{Related work}

We summarize many researches which are related to our research. The surveys related the similarity coefficients to calculate the valences of words are in [1,13-18]. In the research [1], the authors generate several Norwegian sentiment lexicons by extracting sentiment information from two different types of Norwegian text corpus, namely, news corpus and discussion forums. The methodology is based on the Point wise Mutual Information (PMI), etc.

The English dictionaries are [19-21] and there are more than 55,000 English words (including English nouns, English adjectives, English verbs, etc.) from them.

There are the works related to the Dennis Coefficient (DNC) in $[2,3,5]$. The authors in [3] collected 76 binary similarity and distance measures used over the last century and reveal their correlations through the hierarchical clustering technique, etc.

There are the researches related the Latent Semantic Analysis (LSA) in $[4,6,7]$. The study in [4] presents a novel statistical method for factor analysis of binary and count data which is closely related to a technique known as Latent Semantic Analysis, etc.

The latest researches of the sentiment classification are [8-12]. In the research [9], the authors have explored different methods of improving the accuracy of sentiment classification. The authors' proposed method based on the combination of TermCounting method and Enhanced Contextual Valence Shifters method has improved the accuracy of sentiment classification, etc.

\section{Methodology}

Our methodology comprises 3 sub-sections as follows: (1) First sub-section: Creating the sentiment lexicons of the bESD; (2) Second sub-section: Improving the LSA according to the 
sentiment lexicons of the bESD a sequential environment and a distributed network system; (3) Third sub-section: Using the LSA and a DNC to cluster the documents of the testing data set into either the positive or the negative in both a sequential environment and a parallel distributed system.

We built our the testing data set including the 11,000,000 documents in the movie field, which contains the 5,500,000 positive and 5,500,000 negative in English. We also built our the training data set including the 5,000,000 documents in the movie field, which contains the 2,500,000 positive and 2,500,000 negative in English. All the English documents in our testing data set and training data set are automatically extracted from millions of the documents of English Facebook, English websites and social networks; then we labeled positive and negative for them.

\subsection{Creating the sentiment lexicons of the bESD}

\section{Calculating a valence of one word (or one phrase) in English}

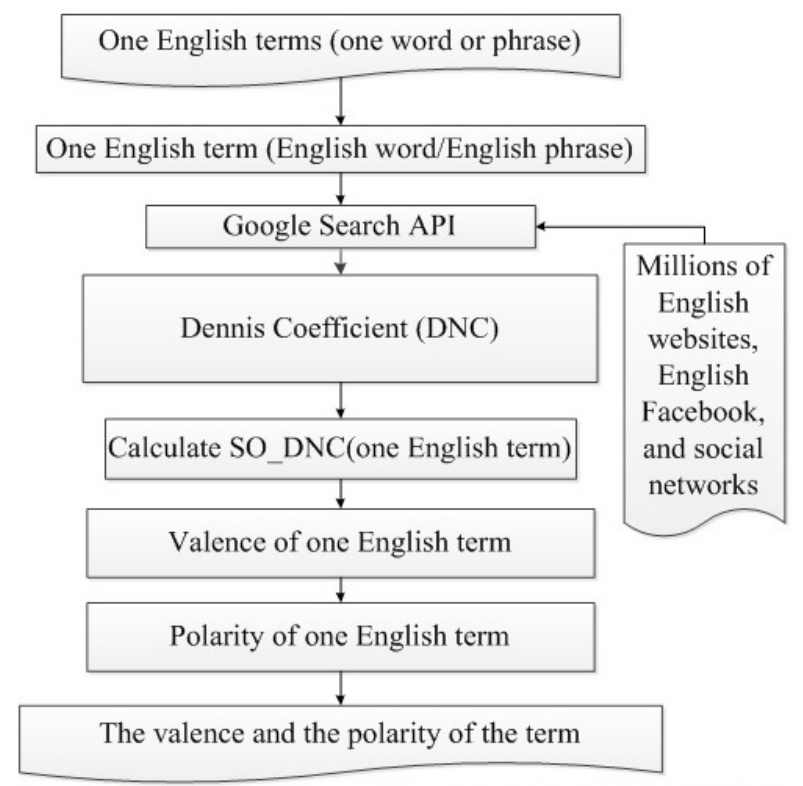

Figure 1: Overview of identifying the valence and the polarity of one term in English using a Dennis coefficient (DNC)

In this part, we calculated the valence and the polarity of one English word (or phrase) by using the DNC through a Google search engine with AND operator and OR operator, as the following diagram in Figure 1 shows.

According to [1,13-18], Pointwise Mutual Information (PMI) between two words wi and wj has the equation

$$
P M I\left(w_{i}, w_{j}\right)=\log _{2}\left[\frac{P\left(w_{i}, w_{j}\right)}{P\left(w_{i}\right) \times P\left(w_{j}\right)}\right]
$$

and $\mathrm{SO}$ (sentiment orientation) of word wi has the equation

$$
S O\left(w_{i}\right)=P M I\left(w_{i}, \text { positive }\right)-P M I\left(w_{i}, \text { negative }\right)
$$

In the research [1], the authors generate several Norwegian sentiment lexicons by extracting sentiment information from two different types of Norwegian text corpus, namely, news corpus and discussion forums. The methodology is based on the Point wise Mutual Information (PMI), 
etc. The authors in [13] used the Ochiai Measure through the Google search engine with AND operator and OR operator to calculate the sentiment values of the words in Vietnamese. The authors in [14] used the Consine Measure through the Google search engine with AND operator and OR operator to identify the sentiment scores of the words in English. The authors in [15] used the Sorensen Coefficient through the Google search engine with AND operator and OR operator to calculate the sentiment values of the words in English. The authors in [16] used the Jaccard Measure through the Google search engine with AND operator and OR operator to calculate the sentiment values of the words in Vietnamese. The authors in [17] used the Tanimoto Coefficient through the Google search engine with AND operator and OR operator to identify the sentiment scores of the words in English.

With the above proofs, we had the information about the measures as follows: PMI was used with AltaVista in English, Chinese, and Japanese with the Google in English; Jaccard was used with the Google in English, Chinese, and Vietnamese. The Ochiai was used with the Google in Vietnamese. The Consine and Sorensen were used with the Google in English.

According to [1,13-18], PMI, Jaccard, Consine, Ochiai, Sorensen, Tanimoto, and DNC were the similarity measures between two words, and they can perform the same functions and with the same chracteristics; so DNC was used in calculating the valence of the words. In addition, we proved that DNC can be used in identifying the valence of the English word through the Google search with the AND operator and OR operator.

With the Dennis coefficient (DNC) in $[2,3,5]$, we had the equation of the DNC:

$$
D N C(a, b)=\frac{[(a \cap b) \times[(\neg a) \cap(\neg b)]-[(\neg a) \cap b] \times[a \cap(\neg b)]]}{\sqrt{n \times[(a \cap b)+[(\neg a) \cap b]] \times[(a \cap b)+[a \cap(\neg b)]]}}
$$

with $\mathrm{a}$ and $\mathrm{b}$ are the vectors.

In this study, we chose $\mathrm{n}=1$. Therefore, we had eq. (4) as follows:

$$
D N C(a, b)=\frac{[(a \cap b) \times[(\neg a) \cap(\neg b)]-[(\neg a) \cap b] \times[a \cap(\neg b)]]}{\sqrt{[(a \cap b)+[(\neg a) \cap b]] \times[(a \cap b)+[a \cap(\neg b)]]}}
$$

From the eq. (1), (2), (3), we proposed many new equations of the DNC to calculate the valence and the polarity of the English words (or the English phrases) through the Google search engine as the following equations below. In eq. (3), when a had only one element, a is a word. When $\mathrm{b}$ had only one element, $\mathrm{b}$ is a word. In eq. (3), a was replaced by w1 and b was replaced by w2.

$$
\text { DennisMeasure }\left(w_{1}, w_{2}\right)=\text { DennisCoefficient }\left(w_{1}, w_{2}\right)=\operatorname{DNC}\left(w_{1}, w_{2}\right)=\frac{B}{\sqrt{A}}
$$

with

(1) $\mathrm{B}=\mathrm{P}\left(w_{1}, w_{2}\right) \times \mathrm{P}\left(\neg w_{1}, \neg w_{2}\right)-\mathrm{P}\left(\neg w 1, w_{2}\right) \times \mathrm{P}\left(w_{1}, \neg w_{2}\right)$;

(2) $\mathrm{A}=\left[\mathrm{P}\left(w_{1}, w_{2}\right)+\mathrm{P}\left(\neg w_{1}, w_{2}\right)\right] \times\left[\mathrm{P}\left(w_{1}, w_{2}\right)+\mathrm{P}\left(w_{1}, \neg w_{2}\right)\right]$.

Eq. (5) was similar to eq. (1). In eq. (2), eq. (1) was replaced by eq. (4). We had eq. (6) as follows:

$$
\operatorname{Valence}(w)=S O \_D N C(w)=D N C(w, \text { positive_query })-D N C(w, \text { negative_query })
$$

In eq. (5), $w_{1}$ was replaced by $\mathrm{w}$ and $w_{2}$ was replaced by position_query. We had eq. (7). Eq. (7) was as follows:

$$
D N C(w, \text { positive_query })=\frac{B 7}{\sqrt{A 7}}
$$


with

(1) $\mathrm{B} 7=\mathrm{P}(\mathrm{w}$, positive_query $) \times \mathrm{P}(\neg w, \neg$ positive_query $) \mathrm{P}(\neg w$,positive_query $)$

$\times \mathrm{P}(\mathrm{w}, \neg$ positive_query $)$;

(2) $\mathrm{A} 7=[\mathrm{P}(\mathrm{w}$, positive_query $)+\mathrm{P}(\neg w$,positive_query $)]$

$\times[\mathrm{P}(\mathrm{w}$, positive_query $)+\overline{\mathrm{P}}(\mathrm{w}, \neg$ positive_query $)]$.

In eq. (5), $w_{1}$ was replaced by $\mathrm{w}$ and $w_{2}$ was replaced by negative_query. We had eq. (8). Eq. (8) was as follows:

$$
D N C(w, \text { negative_query })=\frac{B 8}{\sqrt{A 8}}
$$

with:

(1) $\mathrm{B} 8=\mathrm{P}($ w,negative_query $) \times \mathrm{P}(\neg w, \neg$ negative_query $)-\mathrm{P}(\neg w$, negative_query $)$

$\times \mathrm{P}(\mathrm{w}, \neg$ negative_query $)$;

(2) $\mathrm{A} 8=[\mathrm{P}(\mathrm{w}$, negative_query $)+\mathrm{P}(\neg w$,negative_query $)]$

$\times[\mathrm{P}(\mathrm{w}$, negative_query $)+\overline{\mathrm{P}}(\mathrm{w}, \neg$ negative_query $)]$.

We had the information about $\mathrm{w}, w_{1}, w_{2}$, and etc. as follows:

(1) $\mathrm{w}, w_{1}, w_{2}$ : were the English words (or the English phrases);

(2) $\mathrm{P}\left(w_{1}, w_{2}\right)$ : number of returned results in Google search by keyword ( $w_{1}$ and $\left.w_{2}\right)$. We use the Google Search API to get the number of returned results in search online Google by keyword $\left(w_{1}\right.$ and $\left.w_{2}\right)$;

(3) $\mathrm{P}\left(w_{1}\right)$ : number of returned results in Google search by keyword $w_{1}$. We use the Google Search API to get the number of returned results in search online Google by keyword $w_{1}$;

(4) $\mathrm{P}\left(w_{2}\right)$ : number of returned results in Google search by keyword $w_{2}$. We use the Google Search API to get the number of returned results in search online Google by keyword $w_{2}$;

(5) Valence $(\mathrm{W})=\mathrm{SO} \_\mathrm{DNC}(\mathrm{w})$ : valence of English word (or English phrase) w; is SO of word (or phrase) by using the Dennis coefficient (DNC);

(6) positive_query: active or good or positive or beautiful or strong or nice or excellent or fortunate or correct or superior with the positive_query is the a group of the positive English words;

(7) negative_query: passive or bad or negative or ugly or week or nasty or poor or unfortunate or wrong or inferior with the negative_query is the a group of the negative English words;

(8) $\mathrm{P}(\mathrm{w}$, positive_query): number of returned results in Google search by keyword (positive_query and w). We used the Google Search API to get the number of returned results in search online Google by keyword (positive_query and w);

(9) $\mathrm{P}(\mathrm{w}$, negative_query): number of returned results in Google search by keyword (negative_query and w). We used the Google Search API to get the number of returned results in search online Google by keyword (negative_query and w);

(10) $\mathrm{P}(\mathrm{w})$ : number of returned results in Google search by keyword w. We used the Google Search API to get the number of returned results in search online Google by keyword w;

(11) $\mathrm{P}(\neg w$,positive_query): number of returned results in Google search by keyword ( $\neg w$ and positive_query). We used the Google Search API to get the number of returned results in search online Google by keyword ( $\neg w$ and positive_query);

(12) $\mathrm{P}(\mathrm{w}, \neg$ positive_query): number of returned results in the Google search by keyword ( $\mathrm{w}$ and ( $\neg$ positive_query)). We used the Google Search API to get the number of returned results in search online Google by keyword ( $\mathrm{w}$ and ( $\neg$ positive_query)); 
(13) $\mathrm{P}(\neg w, \neg$ positive_query): number of returned results in the Google search by keyword $(\neg w$ and ( $\neg$ positive_query)). We used the Google Search API to get the number of returned results in search online Google by keyword $((\neg w)$ and ( $\neg$ positive_query));

(14) $\mathrm{P}(\neg w$,negative_query): number of returned results in Google search by keyword ( $\neg w$ and negative_query). We used the Google Search API to get the number of returned results in search online Google by keyword ( $\neg w$ and negative_query);

(15) $\mathrm{P}(\mathrm{w}, \neg$ negative_query $)$ : number of returned results in the Google search by keyword ( $\mathrm{w}$ and ( $\neg$ negative_query)). We used the Google Search API to get the number of returned results in search online Google by keyword ( $\mathrm{w}$ and ( $\neg$ negative_query));

(16) $\mathrm{P}\left(\neg w, \neg n e g a t i v e \_q u e r y\right)$ : number of returned results in the Google search by keyword $(\neg w$ and ( $\neg$ negative_query)). We used the Google Search API to get the number of returned results in search online Google by keyword ( $\neg w$ and ( $\neg$ negative_query)).

We have the information about the DNC as follows: (1) DNC(w, positive_query) $\geq 0$ and $\mathrm{DNC}(\mathrm{w}$, positive_query) $\leq 1$. (2) DNC(w, negative_query) $\geq 0$ and $\mathrm{DNC}$ (w, negative_query) $\leq 1$. (3) If $\mathrm{DNC}(\mathrm{w}$, positive_query $)=0$ and $\mathrm{DNC}\left(\mathrm{w}\right.$, negative_query) $=0$ then $\mathrm{SO} \_-\overline{\mathrm{DNC}}(\mathrm{w})$ $=0$. (4) If DNC ( $\mathrm{w}$, positive_query) $=1$ and DNC ( $\mathrm{w}$, negative_query) $=0$ then SO_DNC (w) $=0$. (5) If DNC ( $\mathrm{w}$, positive_query $)=0$ and DNC (w, negative_query) $=1$ then SO_DNC (w) $=-1$. (6) If DNC (w, positive_query) $=1$ and DNC (w, negative_query) $=1$ then SO_DNC(w) $=0$.

So, SO_DNC $(\mathrm{w}) \geq-1$ and $\mathrm{SO} \_$DNC $(\mathrm{w}) \leq 1$.

The polarity of the English word $\mathrm{w}$ is positive polarity If $\mathrm{SO} \_\mathrm{DNC}(\mathrm{w})<0$. The polarity of the English word $\mathrm{w}$ is negative polarity if $\mathrm{SO}_{-} \mathrm{DNC}(\mathrm{w})<0$. The polarity of the English word $\mathrm{w}$ is neutral polarity if SO_DNC $(\mathrm{w})=0$. In addition, the semantic value of the English word $\mathrm{w}$ is SO_DNC (w). The result of calculating the valence w (English word) is similar to the result of calculating valence w by using AltaVista. However, AltaVista is no longer.

In summary, by using eq. (6), eq. (7), and eq. (8), we identified the valence and the polarity of one word (or one phrase) in English by using the DNC through the Google search engine with AND operator and OR operator.

\section{Creating a basis English sentiment dictionary (bESD) in a sequential environment}

In this part, we calculated the valence and the polarity of the English words or phrases for our bESD by using the DNC in a sequential system in the algorithm 1. According to [19-21], we had at least 55,000 English terms, including nouns, verbs, adjectives, etc.

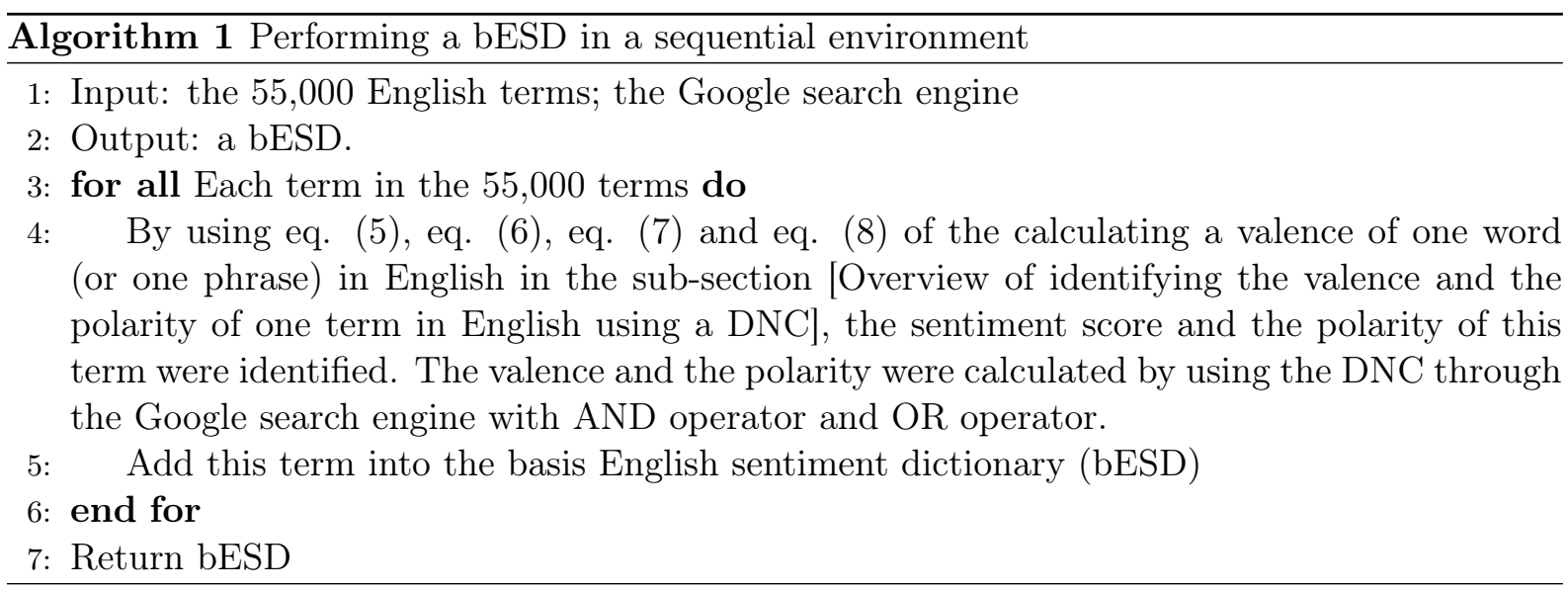

Our basis English sentiment dictionary (bESD) had more 55,000 English words (or English phrases) and bESD was stored in Microsoft SQL Server 2008 R2. 


\section{Creating a basis English sentiment dictionary (bESD) in a distributed system}

In this part, we calculated the valence and the polarity of the English words or phrases for our bESD by using the DNC in a parallel network environment in the algorithm 2 and the algorithm 3. According to [19-21], we had at least 55,000 English terms, including nouns, verbs, adjectives, etc. This section included two phases: the Hadoop Map (M) phase and the Hadoop Reduce (R) phase. The input of the Hadoop Map phase was the 55,000 terms in English in [19-21]. The output of the Hadoop Map phase was one term which the sentiment score and the polarity are identified. The output of the Hadoop Map phase was the input of the Hadoop Reduce phase. Thus, the input of the Hadoop Reduce phase was one term which the sentiment score and the polarity are identified. The output of the Hadoop Reduce phase was the basis English sentiment dictionary (bESD).
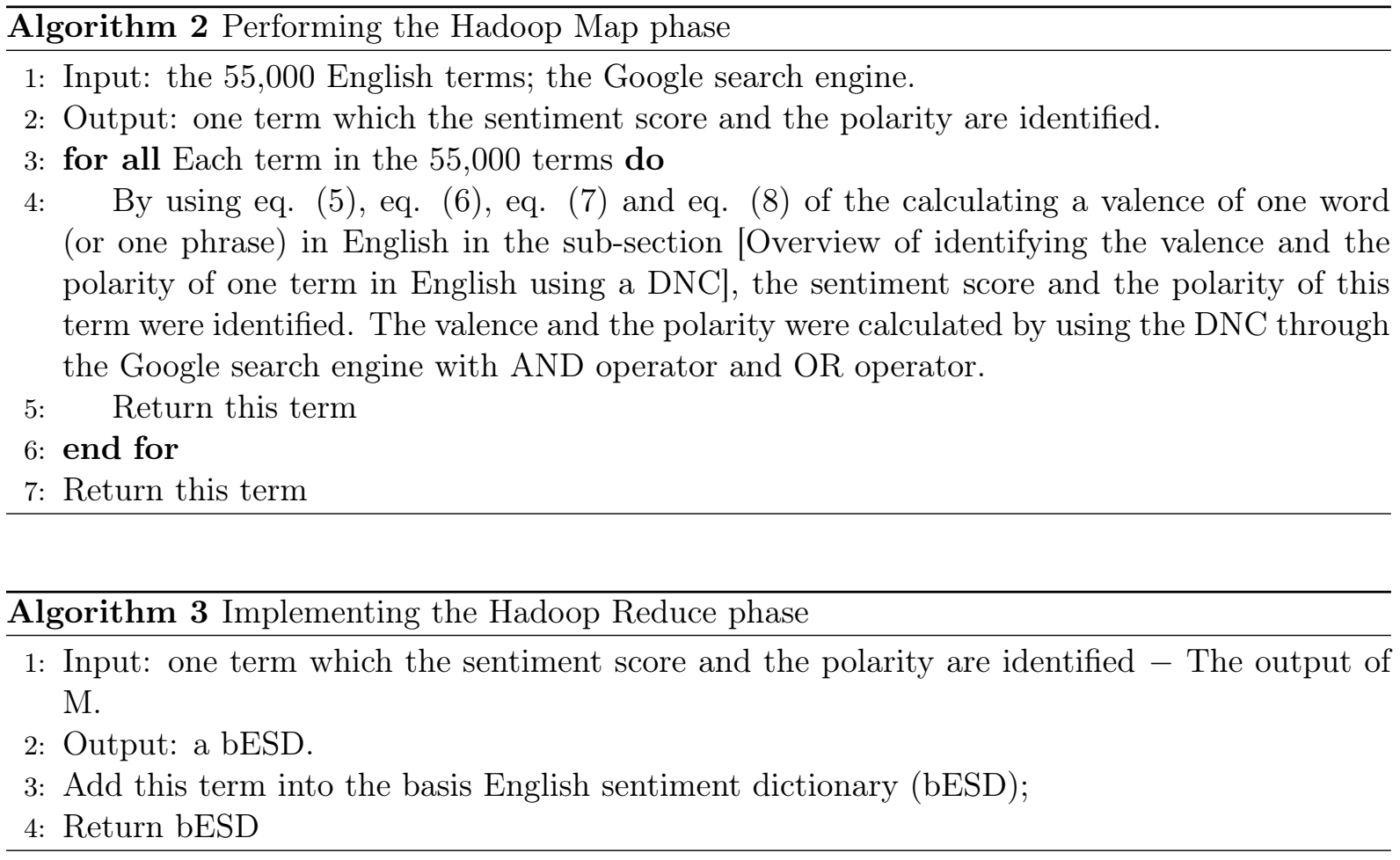

Our bESD had more 55,000 English words (or English phrases) and bESD was stored in Microsoft SQL Server 2008 R2.

\subsection{Improving the LSA according to the sentiment lexicons of the bESD a sequential environment and a distributed network system}

\section{Reforming the LSA based on the sentiment lexicons of the bESD}

According to the Latent semantic analysis (LSA) [4,6,7], it is a technique in natural language processing, that provides a theory and method for extracting and representing the contextualusage and meaning of words by statistical computations applied to a large corpus of text. It closely approximates many aspects of human language learning and understanding. LSA produces a set of concepts (themes) exposed by the document analysis, which are based on the terms contained in the documents. It assumes that words that are similar in meaning occur in similar pieces of texts. We have the basic step involved in LSA based on the LSA $[4,6,7]$ as follows: Computing Term-Passage Matrix -This is the document-term matrix where each row represents 
Table 1: One Latent semantic analysis vector - LSAV

\begin{tabular}{|c|c|c|c|c|c|c|c|}
\hline Documents & Latent & Semantic & Analysis & is & very & useful & slow \\
\hline $\mathrm{d} 1$ & 1 & 1 & 1 & 1 & 2 & 1 & 0 \\
\hline $\mathrm{D} 2$ & 1 & 1 & 1 & 1 & 0 & 0 & 0 \\
\hline
\end{tabular}

a document and the columns represent the terms (occurrence) in the document. Typically, given two documents d1and $\mathrm{d} 2$ with $\mathrm{d} 1=$ "Latent Semantic Analysis is very very useful" and $\mathrm{d} 2=$ "Latent Semantic Analysis is slow". Then, the document-term matrix is as shown below (called one Latent semantic analysis vector - LSAV) in Table $1 . \Rightarrow$ The Latent semantic analysis vector:

$$
L S A V=\left(\begin{array}{lllllll}
1 & 1 & 1 & 1 & 2 & 1 & 0 \\
1 & 1 & 1 & 1 & 0 & 0 & 0
\end{array}\right)
$$

As known, in one sentence, there are sometimes many terms (meaningful words or meaningful phrases) bearing the neutral sentiment, the positive sentiment, or the negative sentiment. For example, we assume that in the bESD, "useful" is the positive sentiment and its valence is +2.3 . "very" is the positive sentiment and its sentiment score is +0.3 . "slow" is the negative sentiment and its valence is -1.1 .

We see that the neutral terms are not the important role in a sentence. Thus, if we still use them in calculating the sentiment of the sentence, there are many noises for this calculating. We also see that the negative terms make many noises for calculating the sentiment of the positive polarity and the positive terms make many noises for calculating the sentiment of the negative polarity. We use the valences of the terms combined with their frequencies to remove many noises of identifying the sentiment classification of one sentence. We apply the valences of the sentiment lexicons of the bESD into the Vd1 and Vd2 as follows: According to the bESD, it is assumed that "Latent" is 0 of its valence; "Semantic" is 0 of its sentiment value; "Analysis" is 0 of its sentiment score; "useful" of +2.3 of its valence; "is" is 0 of its sentiment score; "document" is 0 of its valence; "classification" is 0 of its sentiment value; "an" is 0 of its valence; "very" is +0.3 of its sentiment score; the sentiment value of "slow" is -1.1 . Therefore, we have $\mathrm{d} 1 \mathrm{and} \mathrm{d} 2$ in Table 2.

$\Rightarrow \mathrm{d} 1=(0,0,0,0,0.6,2.3,0)$ and $\mathrm{d} 2=(0,0,0,0,0,0,0)$

$\Rightarrow$ emphasizing on the positive terms and the negative terms in one sentence. $\Rightarrow$ The Latent semantic analysis vector:

$$
L S A V=\left(\begin{array}{lllllll}
1 & 1 & 1 & 1 & 2 & 1 & 0 \\
1 & 1 & 1 & 1 & 0 & 0 & 0
\end{array}\right)
$$

In one LSA vector $(\mathrm{LSAV})$, the value of each element is (its valences) $\times$ (its frequency).

Table 2: The value of $\mathrm{d} 1$ and the value of $\mathrm{d} 2$

\begin{tabular}{|c|c|c|c|c|c|c|c|}
\hline Documents & Latent & Semantic & Analysis & is & very & useful & slow \\
\hline $\mathrm{d} 1$ & $1 \times(0)$ & $1 \times(0)$ & $1 \times(0)$ & $1 \times(0)$ & $2 \times(+0.3)$ & $1 \times(+2.3)$ & $0 \times(-1.1)$ \\
\hline $\mathrm{D} 2$ & $1 \times(0)$ & $1 \times(0)$ & $1 \times(0)$ & $1 \times(0)$ & $0 \times(+0.3)$ & $0 \times(+2.3)$ & $0 \times(-1.1)$ \\
\hline
\end{tabular}

\section{Transferring all the documents of the testing data set and the training data set into the LSAVs in a sequential environmnent}

In this section, we proposed the algorithm 4 , the algorithm 5 , the algorithm 6 , and the algorithm 7 in the sequential system as follows: All the positive documents of the training data 
set were transferred into one LSAV, called the positive LSAV group. All the negative documents of the training data set were transferred into one LSAV, called the negative LSAV group. Each document in the documents of the testing data set was transferred into one LSAV.

We implemented the algorithm 4 to create an order list of the LSAV which comprises all the meaningful terms of both the testing data set and the training data set in the sequential system. We proposed the algorithm 5 to transfer one document of the testing data set into one LSAV in the sequential system.

We built the algorithm 6 to transfer the positive documents of the training data set into one positive LSAV in the sequential system, called the positive LSAV group.

We proposed the algorithm 7 to transfer the negative documents of the training data set into one negative LSAV in the sequential system, called the negative LSAV group.

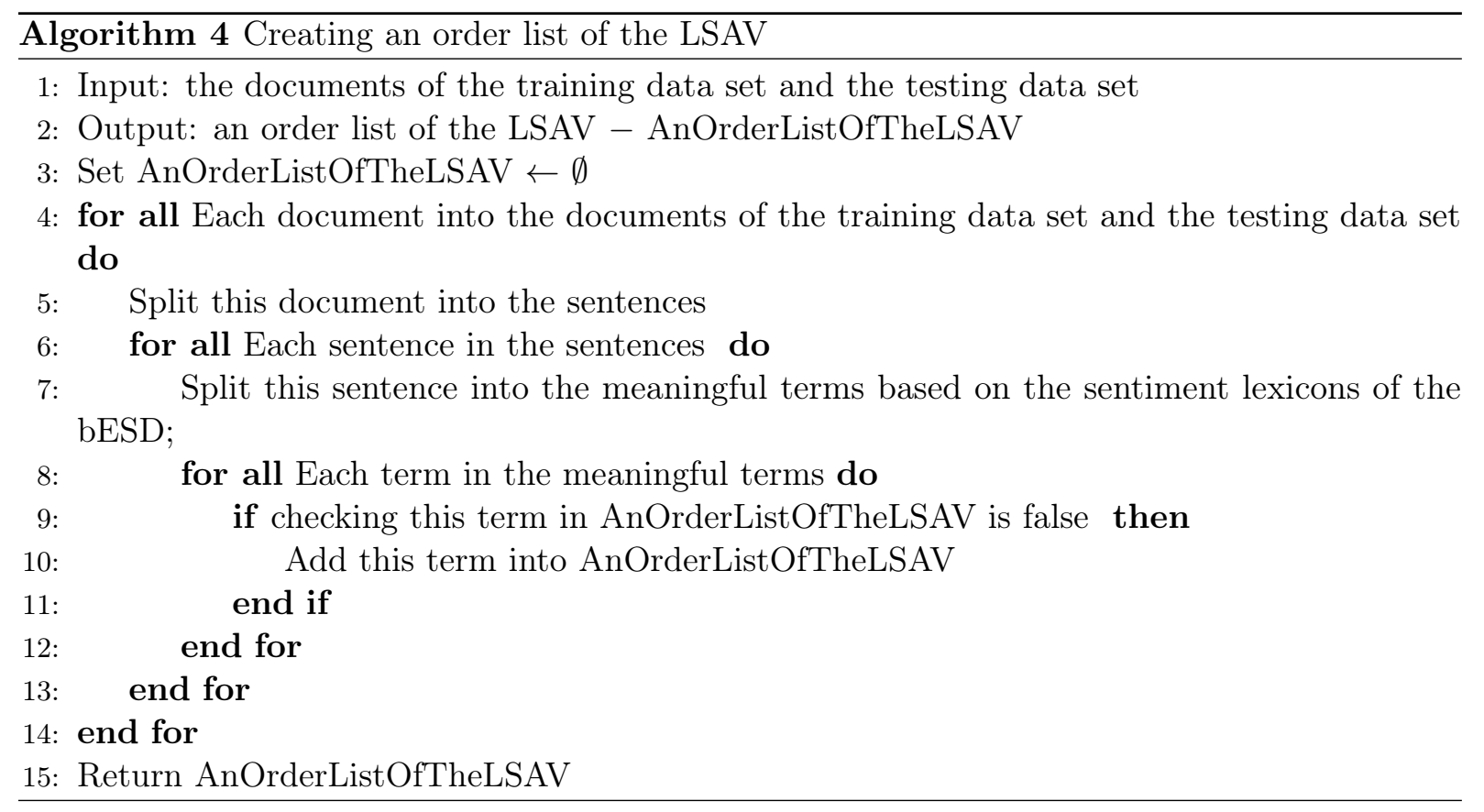

\section{Transferring all the documents of the testing data set and the training data set into the the LSAVs in a distributed network system}

In this section, we implemented many algorithms in the distributed network system as follows: All the positive documents of the training data set were transferred into one LSAV, called the positive LSAV group. All the negative documents of the training data set were transferred into one LSAV, called the negative LSAV group. Each document in the documents of the testing data set was transferred into one LSAV.

This section comprises the algorithm 8, the algorithm 9, the algorithm 10, the algorithm 11, the algorithm 12, the algorithm 13, the algorithm 14, and the algorithm 15.

We created an order list of the LSAV which comprises all the meaningful terms of both the testing data set and the training data set in the distributed network system in the algorithm 8 and the algorithm 9. This stage included two phases: the Hadoop Map phase (M) and the Hadoop Reduce phase $(\mathrm{R})$. The input of $\mathrm{M}$ was the documents of the testing data set and the training data set. The output of $\mathrm{R}$ is one term. The input of $\mathrm{R}$ was the output of $\mathrm{M}$, thus, the input of $\mathrm{R}$ was one term. The output of $\mathrm{R}$ was an order list of the LSAV - AnOrderListOfTheLSAV.

We transferred one document of the testing data set into one LSAV in the parallel system in the algorithm 10 and the algorithm 11. This stage included two phases: the Hadoop Map phase 


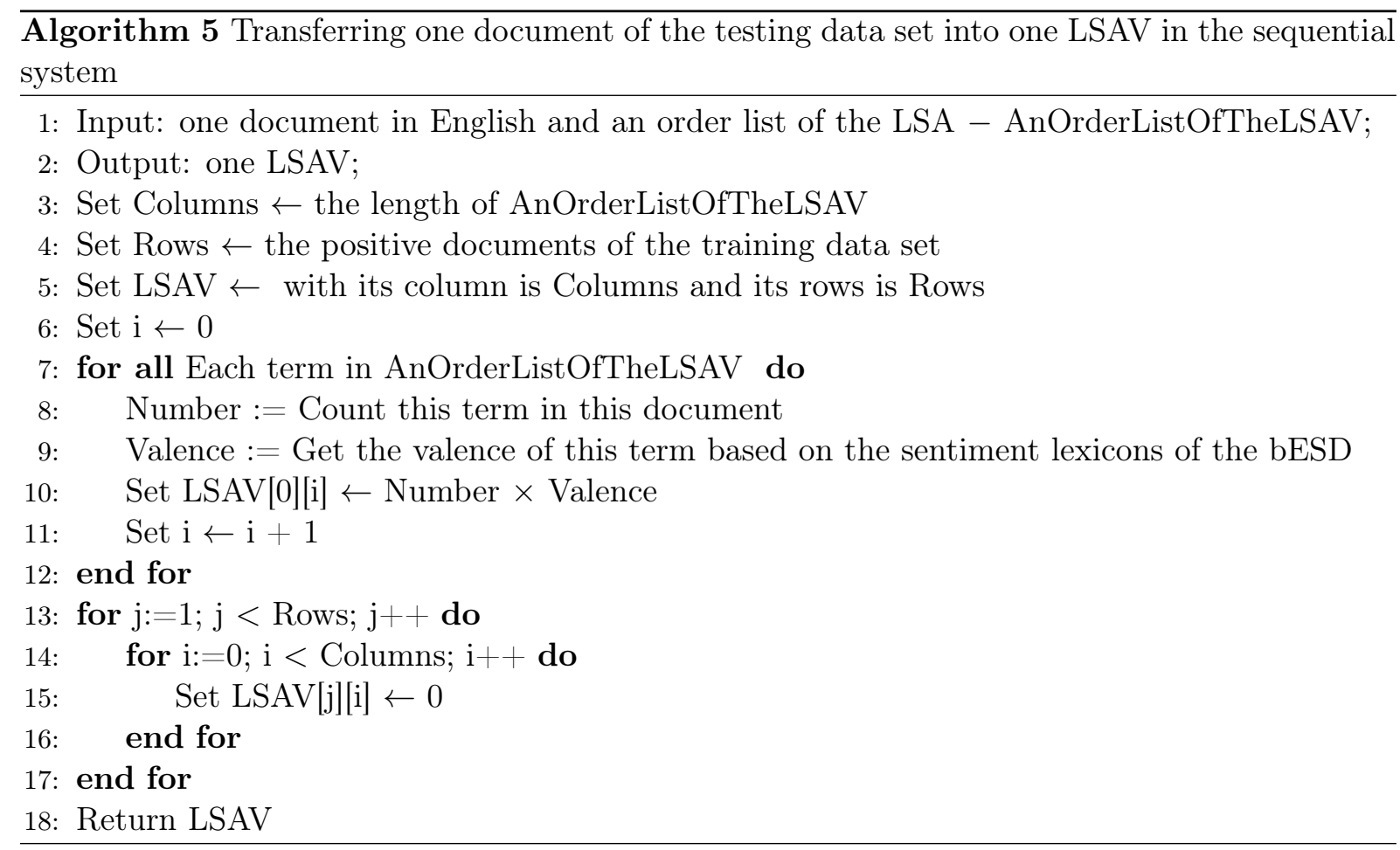

(M) and the Hadoop Reduce phase (R). The input of M was one document in English and an order list of the LSA - AnOrderListOfTheLSAV. The output of M was one row of LSAV. The input of $\mathrm{R}$ was the output of M, thus, the input of $\mathrm{R}$ was one row of LSAV. The output of $\mathrm{R}$ was one LSAV of this document.

We transferred the positive documents of the training data set into one positive LSAV in the distributed system, called the positive LSAV group in the algorithm 12 and the algorithm 13. This stage included two phases: the Hadoop Map phase (M) and the Hadoop Reduce phase (R). The input of $\mathrm{M}$ was the positive documents in English and an order list of the LSA AnOrderListOfTheLSAV. The output of $M$ was one row of PositiveLSAV. The input of $R$ was the output of $\mathrm{M}$, thus, the input of $\mathrm{R}$ was one row of PositiveLSAV. The output of $\mathrm{R}$ was PositiveLSAV.

We transferred the negative documents of the training data set into one negative LSAV in the parallel system, called the negative LSAV group in the algorithm 14 and the algorithm 15 . This stage included two phases: the Hadoop Map phase (M) and the Hadoop Reduce phase $(\mathrm{R})$. The input of the Hadoop Map phase was the negative documents in English and an order list of the LSA - AnOrderListOfTheLSAV. The output of the Hadoop Map phase was one row of NegativeLSAV. The input of the Hadoop Reduce phase was the output of the Hadoop Map, thus, the input of the Hadoop Reduce phase was one row of NegativeLSAV. The output of the Hadoop Reduce phase was NegativeLSAV. 


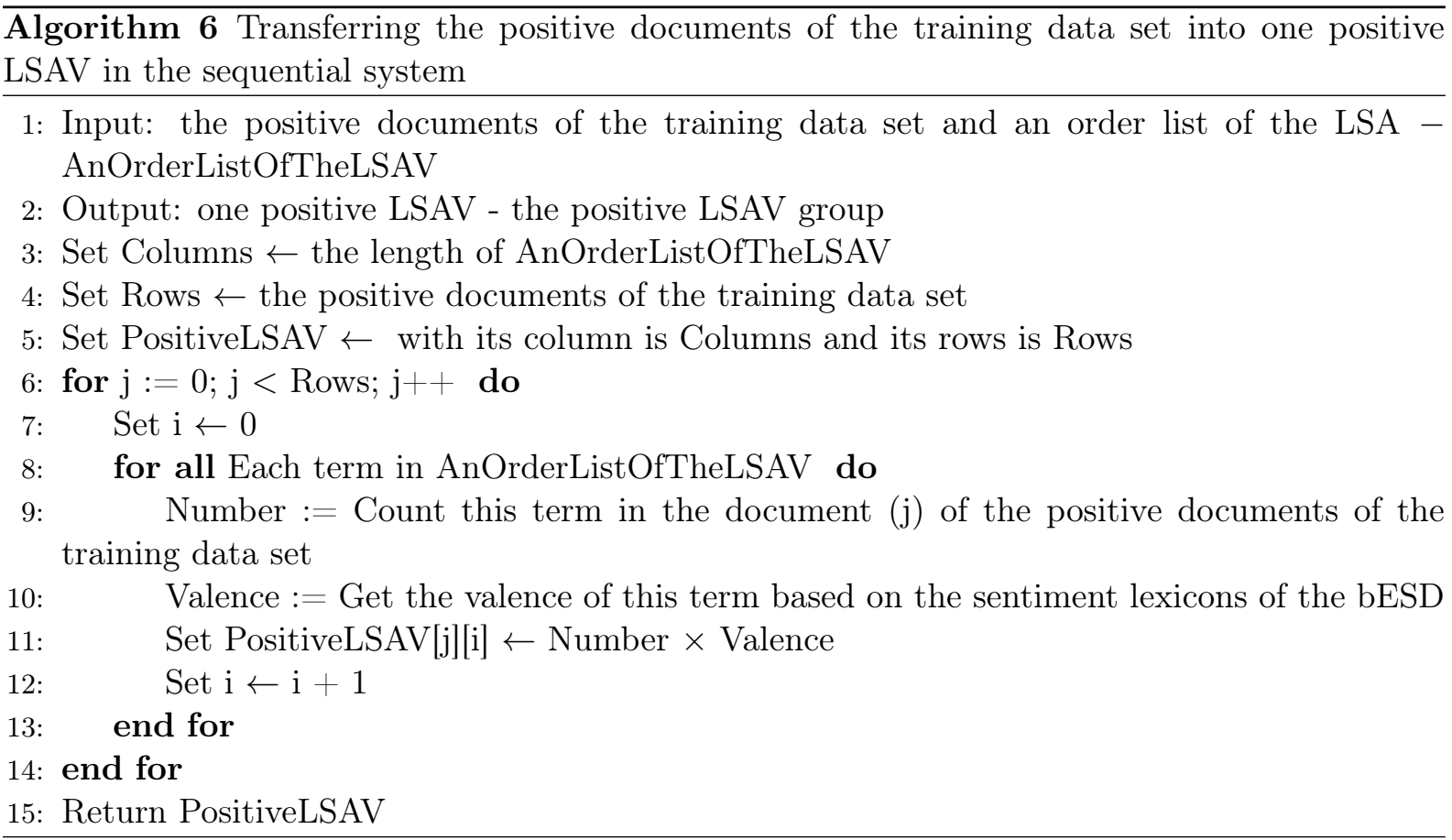

3.3 Using the LSA and a DNC to cluster the documents of the testing data set into either the positive or the negative in both a sequential environment and a parallel distributed system

Using the LSA and a DNC to cluster the documents of the testing data set into either the positive or the negative in a sequential environment

This sub-section has the algorithm 16, and the algorithm 17. In this section, we used the LSA and a DNC to cluster the documents of the testing data set into either the positive or the negative in a sequential environment.

We built the algorithm 16 to cluster one LSAV (corresponding one document of the testing data set) into either positive polarity or the negative polarity in the sequential environment.

We proposed the algorithm 17 to cluster all the documents of the testing data set into either the positive or the negative in the sequential system by using the LSA and the DNC.

Using the LSA and a DNC to cluster the documents of the testing data set into either the positive or the negative in a distributed system

This part includes the algorithm 18, the algorithm 19, the algorithm 20, and the algorithm 21.

In this part, we used the LSA and a DNC to cluster the documents of the testing data set into either the positive or the negative in a distributed system.

We clustered one LSAV (corresponding one document of the testing data set) into either positive polarity or the negative polarity in the distributed environment in the algorithm 18 and the algorithm 19. This stage comprised two phases: the Hadoop Map phase (M) and the Hadoop Reduce phase (R). The input of M was one LSAV (corresponding one document of the testing data set); the positive LSAV group and the negative LSAV group. The output of M was the result of the sentiment classification of one document. The input of $R$ was the output of $M$, thus, the input of $R$ was the result of the sentiment classification of one document. The output of $R$ 


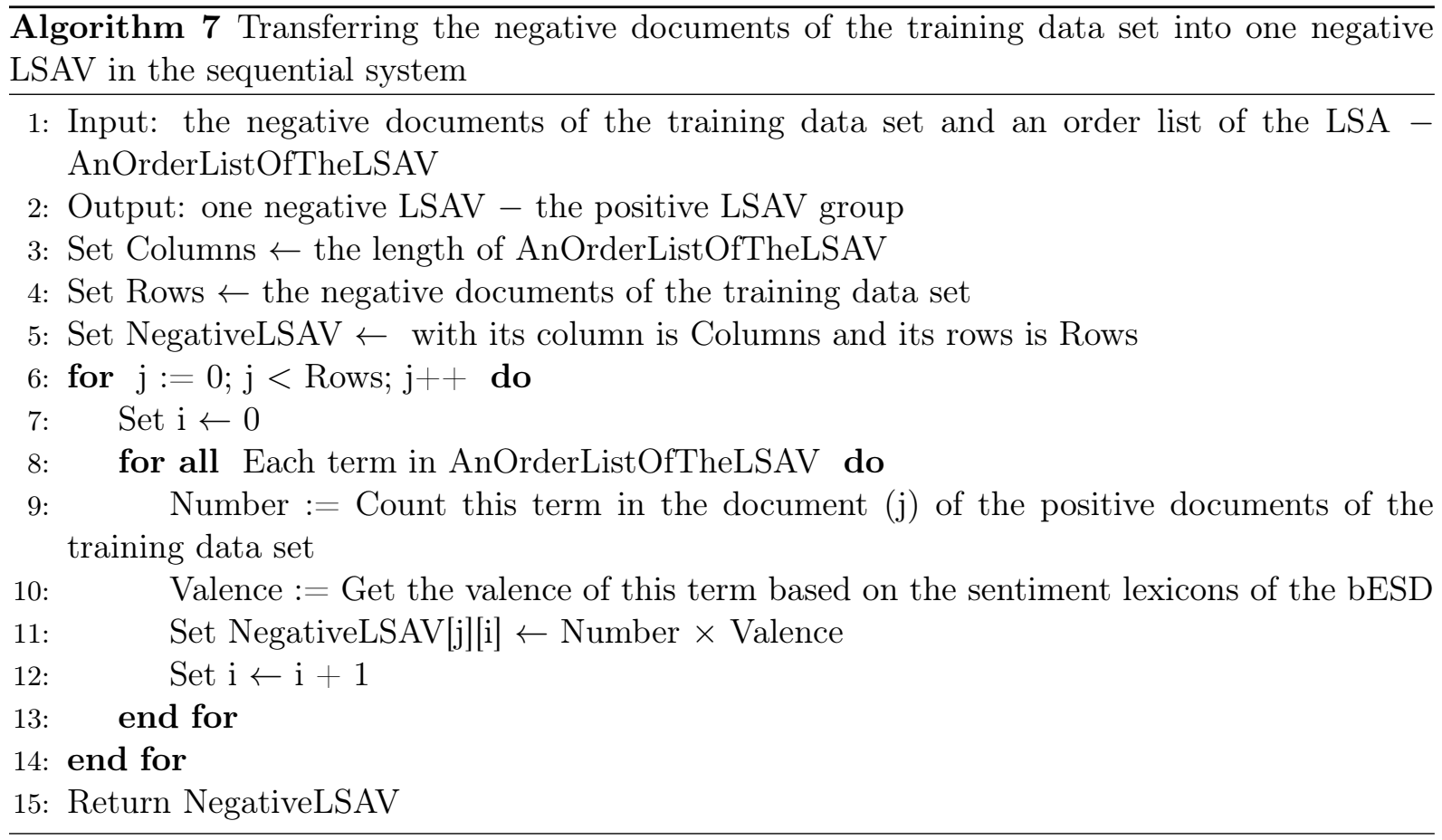

was the result of the sentiment classification of this document of the testing data set.

We used the LSA and the DNC to cluster the documents of the testing data set into either the positive or the negative in the distributed system in the algorithm 20 and the algorithm 21 . This stage comprised two phases: the Hadoop Map phase (M) and the Hadoop Reduce phase (R). The input of $M$ was the documents of the testing data set. The output of $M$ was the result of the sentiment classification of one document. The input of $\mathrm{R}$ was the output of $\mathrm{M}$, thus, the input of $R$ is the result of the sentiment classification of one document. The output of $R$ was the results of the sentiment classification of the documents of the testing data set.

\section{Experiment}

To implement the proposed model, we have already used Java programming language to save the 11,000,000 documents of the testing data set and the 5,000,000 documents of the training data set, and to save the results of emotion classification. The proposed model was implemented in both the sequential system and the distributed network environment.

The configuration of one server is: IntelÂŽ Server Board S1200V3RPS, IntelÂŽ PentiumÂž Processor G3220 (3M Cache, 3.00 GHz), 2GB CC3-10600 ECC 1333 MHz LP Unbuffered DIMMs; and the operating system of the server is: Cloudera.

Our novel model related to the Latent Semantic Analysis and a Dennis Coefficient is implemented in the sequential environment with the configuration as follows: The sequential environment in this research includes 1 node (1 server).

The proposed model related to the Latent Semantic Analysis and a Dennis Coefficient is performed in the Cloudera parallel network environment with the configuration as follows: This Cloudera system includes 9 nodes (9 servers). All 9 nodes have the same configuration information

In Table 3, we show the accuracy and the results of our novel model in the testing data set.

The average time of the classification of our new model for the English documents in testing data set are displayed in Table 4. 


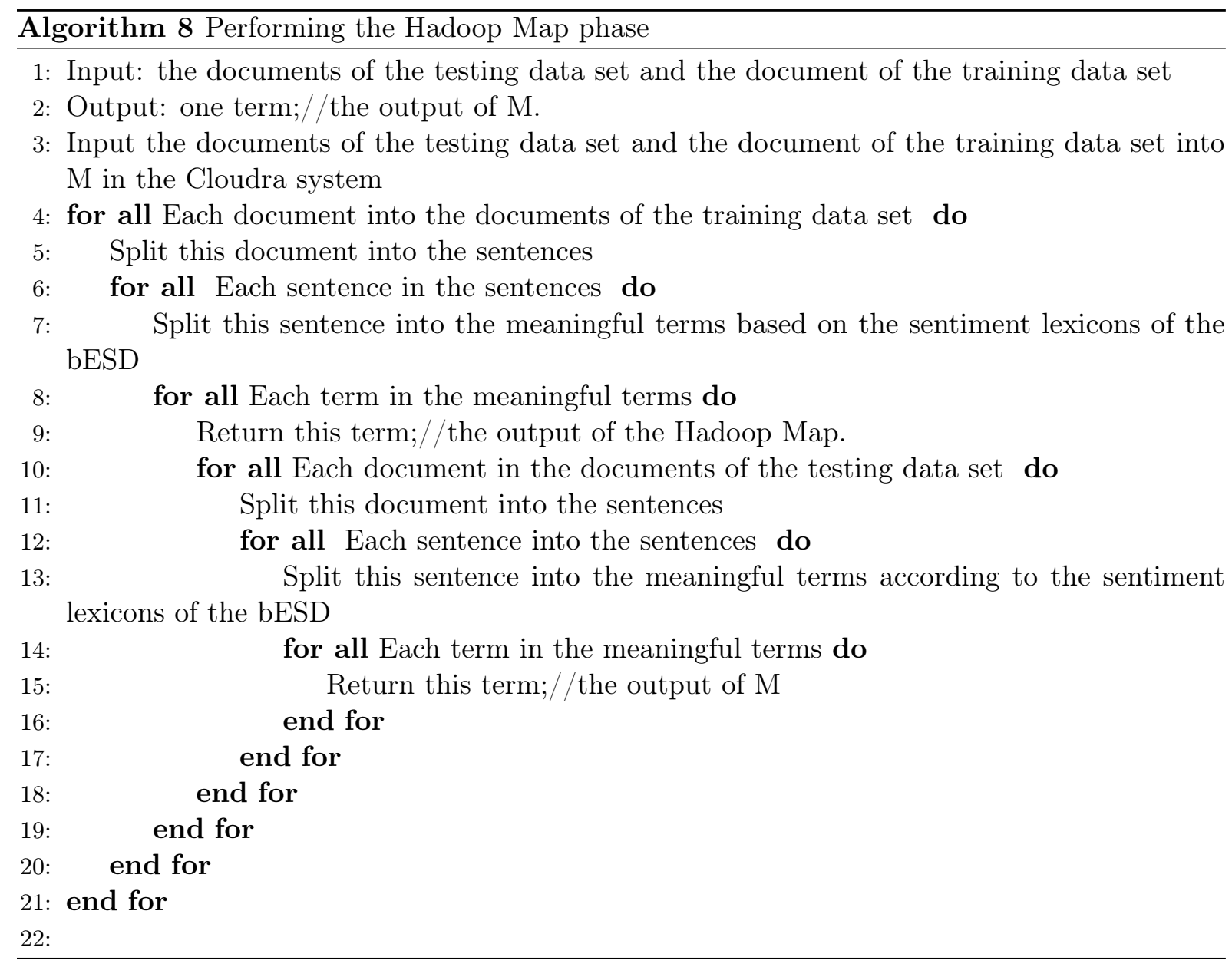

Table 3: The accuracy and The results of our novel model in the testing data set.

\begin{tabular}{|c|c|c|c|c|}
\hline & Testing Dataset & Correct Classification & Incorrect Classification & Accuracy \\
\hline Negative & $5,500,000$ & $4,884,751$ & 615,249 & \\
Positive & $5,500,000$ & $4,878,849$ & 621,151 & $88.76 \%$ \\
Summary & $11,000,000$ & $9,763,600$ & $1,236,400$ & \\
\hline
\end{tabular}

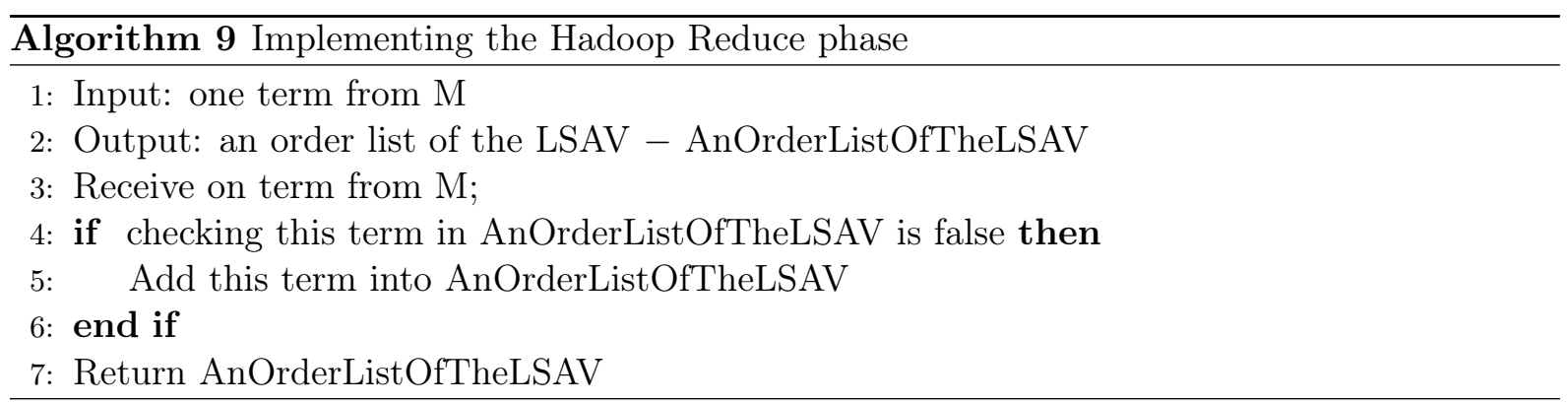



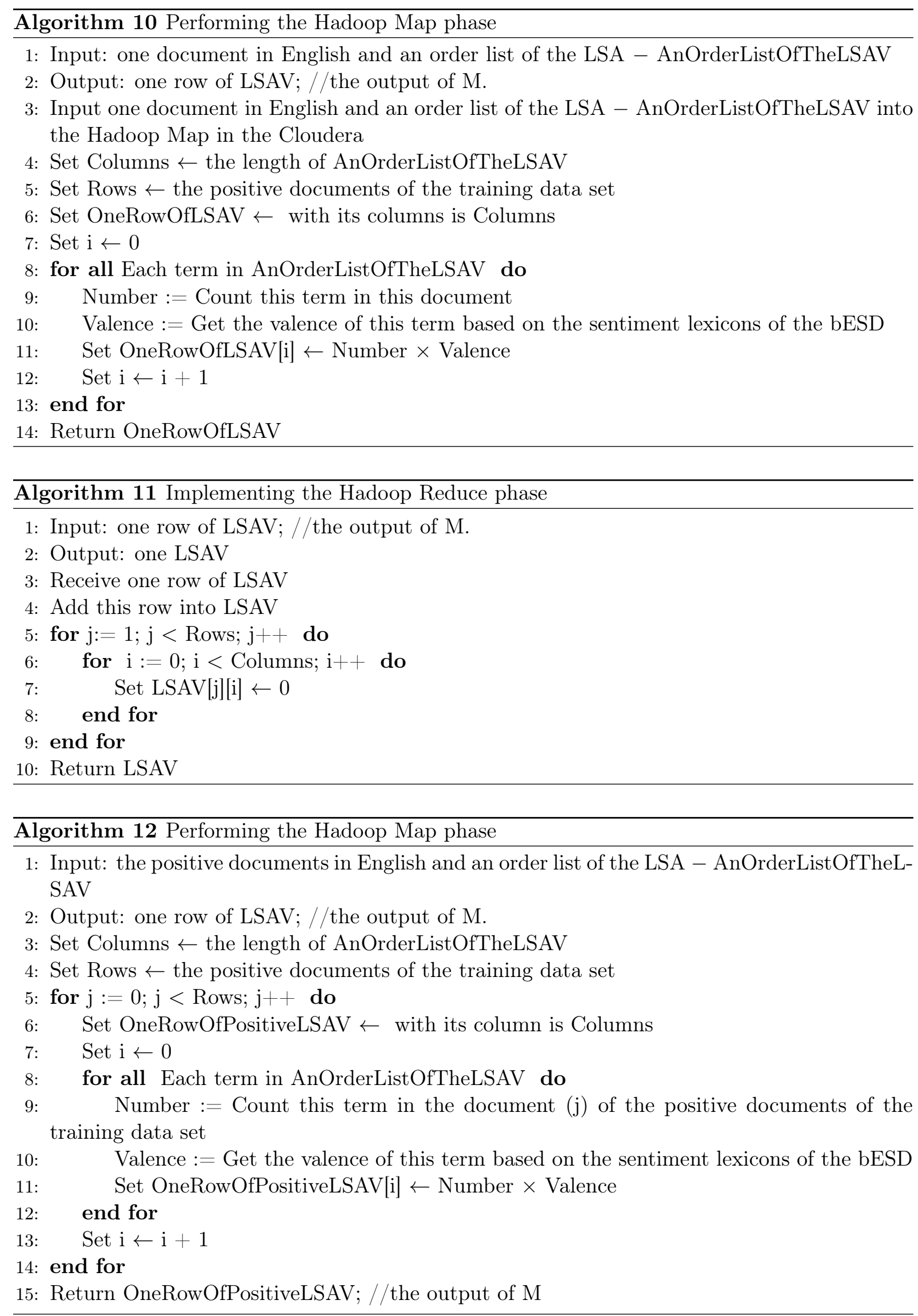

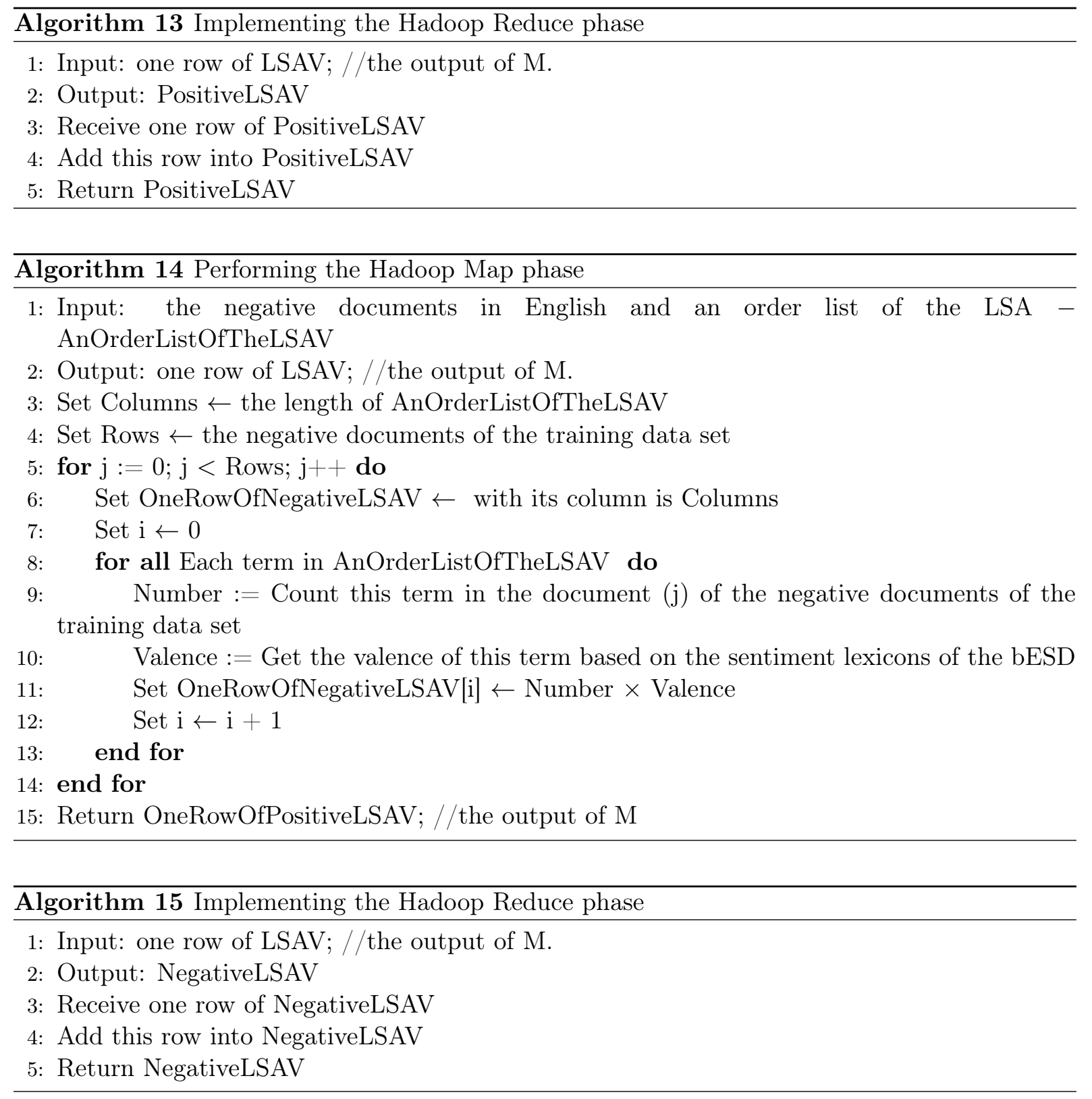

Table 4: Average time of the classification of our new model for the English documents in testing data set

\begin{tabular}{|l|l|}
\hline & $\begin{array}{l}\text { Average time of the classifica- } \\
\text { tion 11,000,000 English docu- } \\
\text { ments. }\end{array}$ \\
\hline $\begin{array}{l}\text { The Latent Semantic Analysis and a Dennis Coefficient } \\
\text { in the sequential environment }\end{array}$ & $43,460,194$ seconds \\
\hline $\begin{array}{l}\text { The Latent Semantic Analysis and a Dennis Coefficient } \\
\text { in the Cloudera distributed system with 3 nodes }\end{array}$ & $13,120,064$ seconds \\
\hline $\begin{array}{l}\text { The Latent Semantic Analysis and a Dennis Coefficient } \\
\text { in the Cloudera distributed system with 6 nodes }\end{array}$ & $7,360,032$ seconds \\
\hline $\begin{array}{l}\text { Latent Semantic Analysis and a Dennis Coefficient in } \\
\text { the Cloudera distributed system with 9 nodes }\end{array}$ & $4,851,132$ seconds \\
\hline
\end{tabular}




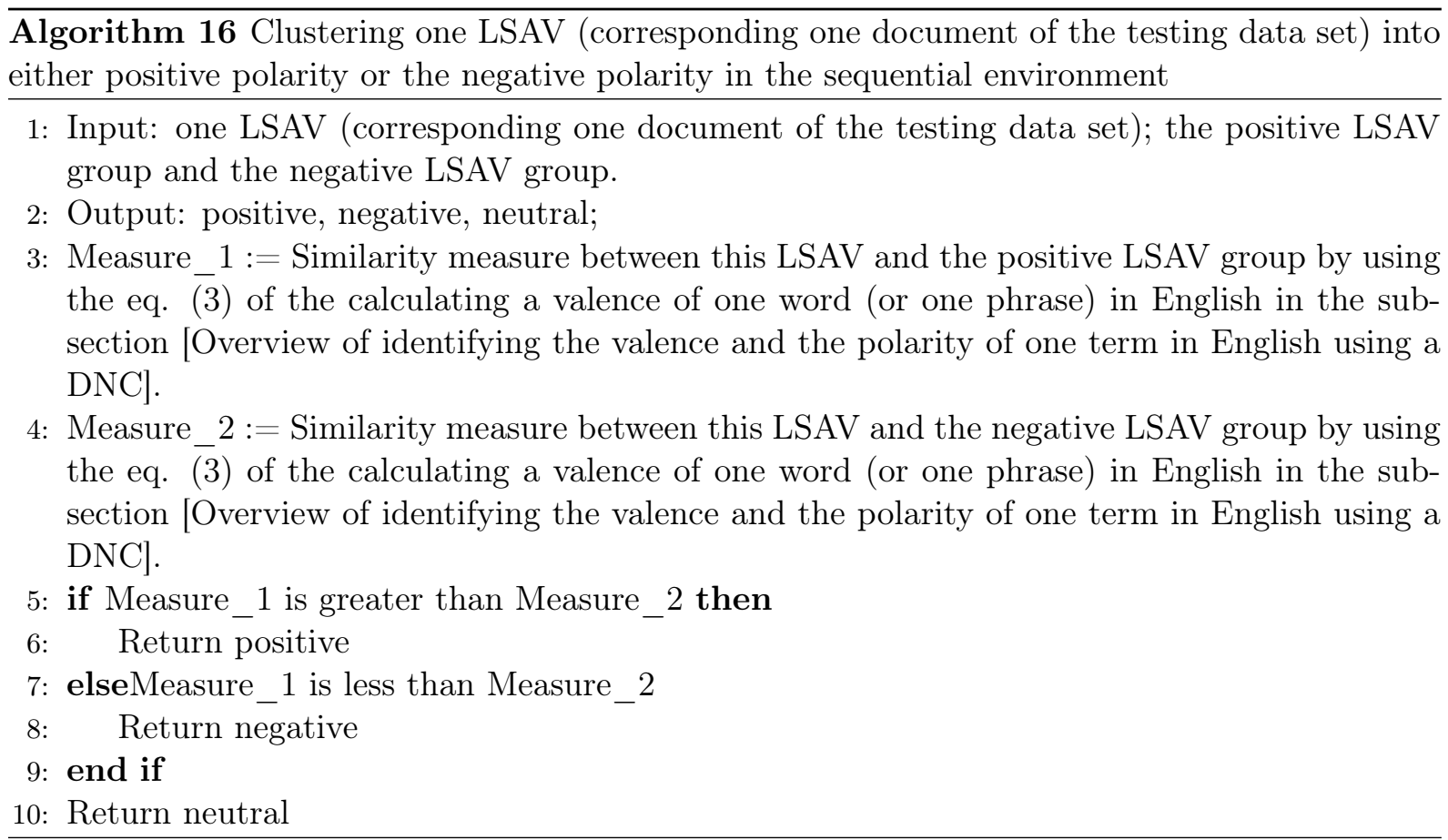

Algorithm 17 Clustering all the documents of the testing data set into either the positive or the negative in the sequential system by using the LSA and the DNC

1: Input: the documents of the testing data set and the training data set.

2: Output: positive, negative, neutral;

3: the creating a bESD in a sequential environment in the sub-section [Creating a basis English sentiment dictionary (bESD) in a sequential environment].

4: the algorithm 4 to create an order list of the LSAV which comprises all the meaningful terms of both the testing data set and the training data set in the sequential system.

5: the algorithm 6 to transfer the positive documents of the training data set into one positive LSAV in the sequential system, called the positive LSAV group.

6: the algorithm 7 to transfer the negative documents of the training data set into one negative LSAV in the sequential system, called the negative LSAV group.

7: Results := null;

8: for all Each document in the documents of the testing data set do

9: One LSAV $:=$ the algorithm 5 to transfer one document of the testing data set into one LSAV in the sequential system.

10: OneResult $:=$ the algorithm 16 to cluster one LSAV (corresponding one document of the testing data set) into either positive polarity or the negative polarity in the sequential environment.

11: $\quad$ Add OneResult into Results;

12: end for

13: Return Results; 

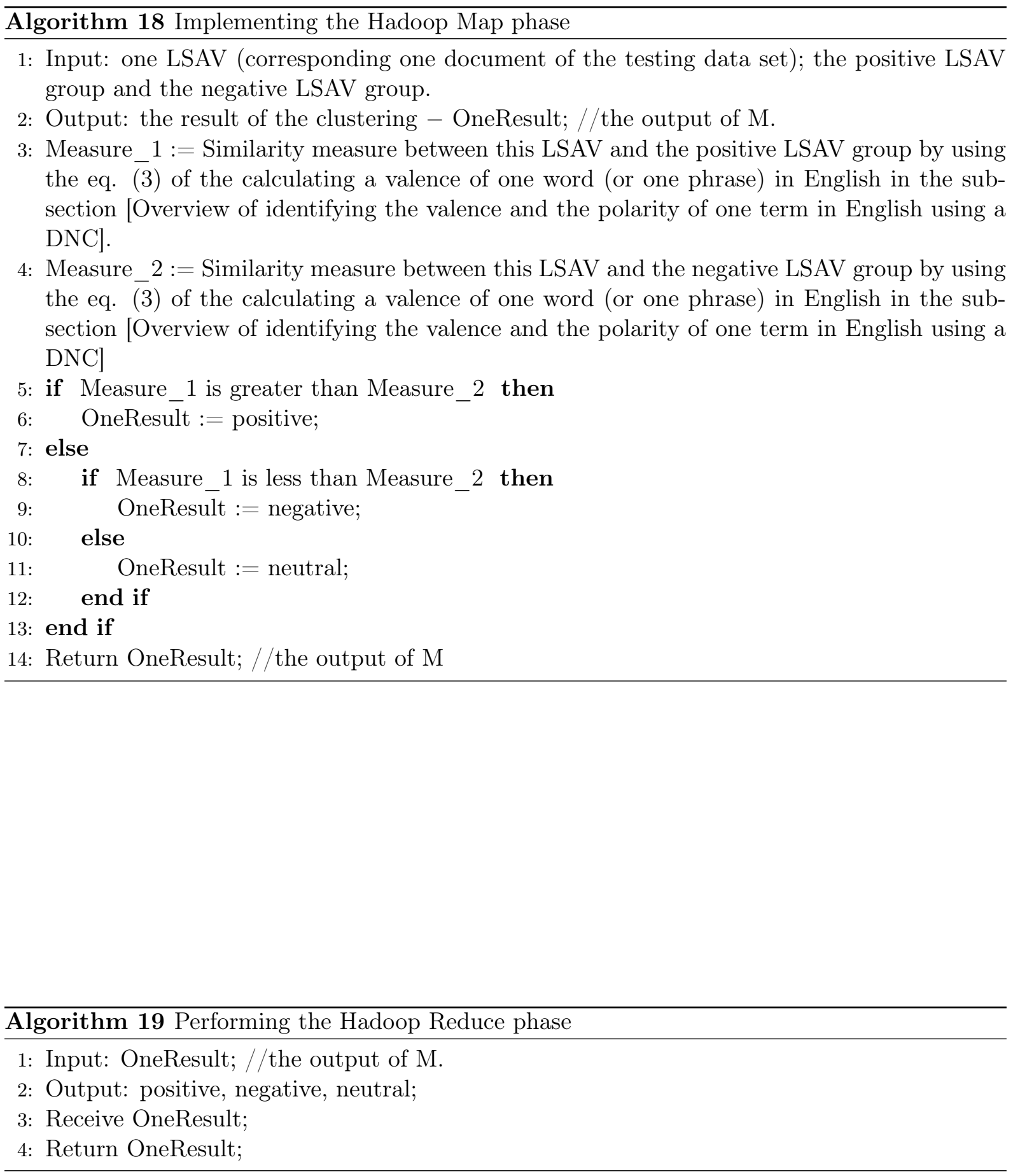

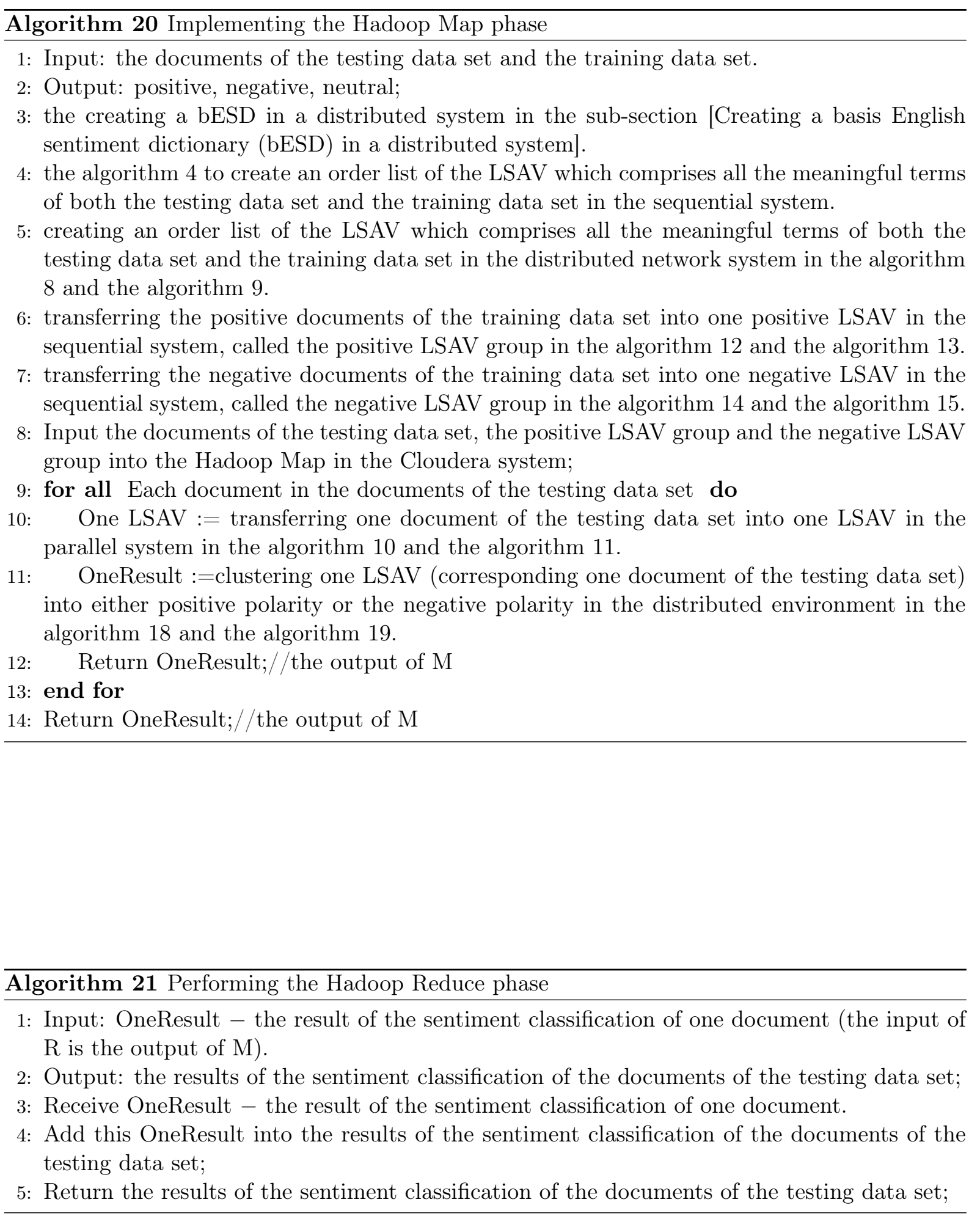


\section{Conclusion}

In this survey, a new model has been proposed to classify sentiment of many documents in English using the Latent Semantic Analysis and a Dennis Coefficient with Hadoop Map (M) /Reduce (R) in the Cloudera parallel network environment. Based on our proposed new model, we have achieved $88.76 \%$ accuracy of the testing data set in Table 3, and this is better than the accuracies of many previous models of the semantic analysis. Besides, we have also compared the novel model with the previous models, and the experiments and the results of our proposed model are better than that of the previous model. Until now, not many studies have shown that the clustering methods can be used to classify data. According to Table 4, the average time of the sentiment classification of using the Latent Semantic Analysis and a Dennis Coefficient in the sequential environment is 43,460,194 seconds / 11,000,000 English documents and it is greater than the average time of the sentiment classification of using the Latent Semantic Analysis and a Dennis Coefficient in the Cloudera parallel network environment with 3 nodes which is 13,120,064 seconds / 11,000,000 English documents. The average time of the sentiment classification of using the Latent Semantic Analysis and a Dennis Coefficient in the Cloudera parallel network environment with 9 nodes is 4,851,132 seconds / 11,000,000 English documents, and It is the shortest time in the table. Besides, the average time of the sentiment classification of using the Latent Semantic Analysis and a Dennis Coefficient in the Cloudera parallel network environment with 6 nodes is 7,360,032 seconds / 11,000,000 English documents

The accuracy of the proposed model is dependent on many factors as follows: (1) The LSA related algorithms. (2) The testing data set. (3) The documents of the testing data set must be standardized carefully. (4) Transferring one document into one LSAV.

Table 5: Comparisons of our model's positives and negatives the surveys related to the Latent Semantic Analysis (LSA)

\begin{tabular}{|c|c|c|c|}
\hline Studies & Approach & Positives & Negatives \\
\hline [4] & $\begin{array}{l}\text { Unsupervised } \\
\text { Learning by } \\
\text { Probabilistic } \\
\text { Latent Semantic } \\
\text { Analysis }\end{array}$ & $\begin{array}{l}\text { The survey presents perplexity results for dif- } \\
\text { ferent types of text and linguistic data collec- } \\
\text { tions and discusses an application in automated } \\
\text { document indexing. The experiments indicate } \\
\text { substantial and consistent improvements of the } \\
\text { probabilistic method over standard Latent Se- } \\
\text { mantic Analysis. }\end{array}$ & $\begin{array}{l}\text { No men- } \\
\text { tion }\end{array}$ \\
\hline [6] & $\begin{array}{l}\text { The latent seman- } \\
\text { tic analysis theory } \\
\text { of acquisition, in- } \\
\text { duction, and } \\
\text { representation of } \\
\text { knowledge. }\end{array}$ & $\begin{array}{l}\text { A new general theory of acquired similarity and } \\
\text { knowledge representation, latent semantic anal- } \\
\text { ysis (LSA), is presented and used to successfully } \\
\text { simulate such learning and several other psy- } \\
\text { cholinguistic phenomena. }\end{array}$ & $\begin{array}{l}\text { No men- } \\
\text { tion }\end{array}$ \\
\hline $\begin{array}{l}\text { Our } \\
\text { work }\end{array}$ & $\begin{array}{lll}\text { LSA } & \text { using } & \text { A } \\
\text { DNC } & & \end{array}$ & \multicolumn{2}{|c|}{$\begin{array}{l}\text { The positives and negatives of the proposed model are given } \\
\text { in the Conclusion section. }\end{array}$} \\
\hline
\end{tabular}

The execution time of the proposed model is dependent on many factors as follows: (1) The parallel network environment such as the Cloudera system. (2) The distributed functions such as Hadoop Map (M) and Hadoop Reduce (R). (3) The LSA - related algorithms. (4) The performance of the distributed network system. (5) The number of nodes of the parallel network environment. (6) The performance of each node (each server) of the distributed environment. (7) The sizes of the training data set and the testing data set. (8) Transferring one document into one LSAV. 
The proposed model has many advantages and disadvantages. Its positives are as follows: It uses the Latent Semantic Analysis and a Dennis Coefficient to classify semantics of English documents based on sentences. The proposed model can process millions of documents in the shortest time. This study can be performed in distributed systems to shorten the execution time of the proposed model. It can be applied to other languages. Its negatives are as follows: It has a low rate of accuracy. It costs too much and takes too much time to implement this proposed model.

To understand the scientific values of this research, we have compared our model's results with many studies in the tables below. Our novel model has more benefits than the studies in the tables, and the results of this model are better than that of the works in the tables.

In Table 5, we present the comparisons of our model's positives and negatives the surveys related to the Latent Semantic Analysis (LSA).

The comparisons of our model's benefits and drawbacks with the studies related to the DENNIS coefficient (DNC) are shown in Table 6.

Table 6: Comparisons of our model's benefits and drawbacks with the studies related to the DENNIS coefficient (DNC)

\begin{tabular}{|l|l|l|l|}
\hline Studies & Approach & Benefits & Drawbacks \\
\hline Analysis of & $\begin{array}{l}\text { Macromolecular } \\
\text { Polydispersity } \\
\text { in Intensity } \\
\text { Correlation Spec- } \\
\text { troscopy The } \\
\text { Method of Cumu- } \\
\text { lants }\end{array}$ & $\begin{array}{l}\text { A method is described by which the distribution } \\
\text { function of the decay rates (and thus the extent } \\
\text { of polydispersity) can be characterized, in a light } \\
\text { scattering experiment, by calculation of the mo- } \\
\text { ments or cumulants. }\end{array}$ & $\begin{array}{l}\text { No men- } \\
\text { tion }\end{array}$ \\
\hline 3$]$ & $\begin{array}{l}\text { A Survey of Bi- } \\
\text { nary Similarity } \\
\text { and Distance } \\
\text { Measures }\end{array}$ & $\begin{array}{l}\text { The authors collected 76 binary similarity and } \\
\text { distance measures used over the last century and } \\
\text { reveal their correlations through the hierarchical } \\
\text { clustering technique }\end{array}$ & $\begin{array}{l}\text { No men- } \\
\text { tion }\end{array}$ \\
\hline $\begin{array}{l}\text { Our } \\
\text { work }\end{array}$ & $\begin{array}{l}\text { LSA using A } \\
\text { DNC }\end{array}$ & $\begin{array}{l}\text { The advantages and disadvantages of this survey are shown } \\
\text { in the Conclusion section. }\end{array}$ \\
\hline
\end{tabular}

\section{Conflict of interests}

The authors declare that there is no conflict of interests.

\section{Bibliography}

[1] Bai, A.; Hammer, H.; Yazidi, A.; Engelstad, P. (2014); Constructing sentiment lexicons in Norwegian from a large text corpus, 2014 IEEE 17th International Conference on Computational Science and Engineering, 231-237, 2014.

[2] Baldocchi, D.D.; Hincks, B.B.; Meyers, T.P.(1988); Measuring Biosphere-Atmosphere Exchanges of Biologically Related Gases with Micrometeorological Methods, Ecology society of America, 59(5), 1331-1340, 1988.

[3] Choi, S.-S; Cha, S.-H.; Tappert, C.C. (2010); A Survey Of Binary Similarity And Distance Measures, Systemics, Cybernetics And Informatics, 8(1), 43-48, 2010. 
[4] Hofmann, T. (2001); Unsupervised Learning by Probabilistic Latent Semantic Analysis, Machine Learning, 42(1-2), 177-196, 2001.

[5] Koppel, D.E. (1972); Analysis of Macromolecular Polydispersity in Intensity Correlation Spectroscopy: The Method of Cumulants, The Journal of Chemical Physics, 57(11), 4814, 1972.

[6] Landauer, T.K.; Dumais, S. T. (1997); A solution to Plato's problem: The latent semantic analysis theory of acquisition, induction, and representation of knowledge, Psychological Review, 104(2), 211-240, 1997.

[7] Landauer, T.K.; Foltz, P. W.; Laham, D. (2009); An introduction to latent semantic analysis, Discourse Processes, 25(2-3), 259-284, 2009.

[8] Ngoc, P.V.; Ngoc, C.V.T.; Ngoc, T.V.T. et al. (2017); A C4.5 algorithm for english emotional classification, Evolving Systems, 1-27, 2017.

[9] Phu, V.N. ; Tuoi, P.T. (2014); Sentiment classification using Enhanced Contextual Valence Shifters, International Conference on Asian Language Processing (IALP), 224-229, 2014.

[10] Phu, V.N.; Dat, N.D.; Tran, D.T.N.; Chau, V.T.N.; Nguyen, T.A.(2017); Fuzzy C-Means for English Sentiment Classification in a Distributed System, International Journal of Applied Intelligence, 45(3), 717-738 2017.

[11] Phu, V.N.; Chau, V.T.N.; Tran, D.T.N. (2017); SVM for English Semantic Classification in Parallel Environment, International Journal of Speech Technology, 20(3), 487-508, 2017.

[12] Phu, V.N.; Tran, V.T.N.; Chau, V.T.N. et al. (2017); A Decision Tree using ID3 Algorithm for English Semantic Analysis, International Journal of Speech Technology, 20(3), 593-613, 2017.

[13] Phu, V.N.; Chau, V.T.N.; Tran, V.T.N. et al. (2017); A Vietnamese adjective emotion dictionary based on exploitation of Vietnamese language characteristics, International Journal of Artificial Intelligence Review (AIR), 1-67, 2017

[14] Phu, V.N., Chau, V.T.N., Dat, N.D. et al. (2017); A Valences-Totaling Model for English Sentiment Classification, International Journal of Knowledge and Information Systems, 53(3), 579-636, 2017.

[15] Phu, V.N.; Chau, V.T.N.; Tran, V.T.N(2017); Shifting Semantic Values of English Phrases for Classification, International Journal of Speech Technology, 20(3), 579-636, 2017.

[16] Phu, V.N., Chau, V.T.N., Tran, V.T.N. et al. (2017); A Valence-Totaling Model for Vietnamese Sentiment Classification, International Journal of Evolving Systems, 1-47, 2017.

[17] Phu, V.N., Tran, V.T.N., Chau, V.T.N. et al. (2017); Semantic Lexicons of English Nouns for Classification, International Journal of Evolving Systems, 1-69, 2017.

[18] Turney, D. P.; Littman, M.L. (2002); Unsupervised Learning of Semantic Orientation from a Hundred-Billion-Word Corpus, arXiv:cs/0212012, Learning, 2002.

[19] Cambridge English Dictionary (2017); http://dictionary.cambridge.org/

[20] Longman English Dictionary (2017); http://www.ldoceonline.com/

[21] Oxford English Dictionary (2017); http://www.oxforddictionaries.com/ 